\title{
Comparison of Atorvastatin and Rosuvastatin in Reduction of Inflammatory Biomarkers in Patients with Acute Coronary Syndrome
}

Besham Kumar, Mir Ali Asghar Shah, Rajesh Kumar, Jai Kumar, Aurangzeb Memon

Corresponding author: Besham Kumar, beshamkumar916@gmail.com

\author{
Abstract \\ Introduction \\ High-sensitivity C-reactive protein (hs-CRP) has emerged to be a very useful and reliable clinical marker of \\ primary as well as secondary cardiovascular morbidity and mortality. Elevated hs-CRP contributes to \\ underlying atherogenesis and worsens disease prognosis. Along with their lipid-lowering properties, statins \\ also contribute to the alleviation of micro-inflammation and reduces pro-inflammatory markers. The aim of \\ this study is to compare the effects of rosuvastatin and atorvastatin in lowering hs-CRP levels in statin-naive \\ patients admitted with acute coronary syndrome (ACS).
}

\section{Methods}

In this prospective, open-label randomized trial, group A was given rosuvastatin $40 \mathrm{mg}$ daily and group B was given atorvastatin $20 \mathrm{mg}$ daily along with standard post-ACS therapy. Lipid profile (mg/dL), hs-CRP $(\mathrm{mg} / \mathrm{L})$ and erythrocyte sedimentation rate (ESR) $(\mathrm{mm} / \mathrm{Hr})$ were recorded and measured as the baseline (before starting therapy) and then again after four weeks. The data were analyzed using SPSS for Windows version 22.0 (IBM Corp., Armonk, NY).

\section{Results}

With four weeks of treatment, both group A and B showed statistically significant reduction in serum hsCRP levels ( $\mathrm{p}<0.0001$ ). In group A, there was a mean $51 \%$ decrease in hs-CRP levels, and in group B, a 35\% reduction was seen. Group A showed markedly low hs-CRP levels than group B after four weeks of therapy $(18.46 \pm 6.35$ vs. $24.67 \pm 8.45)(\mathrm{p}<0.0001)$. Group A showed mean $16 \%$ decrease in ESR levels as compared to $14 \%$ decrease in group B. Group A showed lower ESR levels than group B after four weeks of therapy $(19.59 \pm$ 11.83 vs. $20.52 \pm 12.13)(\mathrm{p}<0.0001)$.

\section{Conclusion}

Received 05/24/2019 Review began 05/28/2019 Review ended 06/202/2019 Published 06/14/2019

๑ Copyright 2019 Kumar et al. This is an open access article distributed under the terms of the Creative Commons Attribution License CC-BY 3.0 , which permits unrestricted we distiblon and reprod a

Rosuvastatin showed a 50\% decrease and atorvastatin showed a $35 \%$ reduction in serum hs-CRP levels in statin-naive ACS patients. Rosuvastatin has a more effective role in reducing micro-inflammation in ACS patients.

Categories: Cardiology, Family/General Practice, Internal Medicine

Keywords: hmg-coa reductase inhibitors, atorvastatin, rosuvastatin, high sensitivity c-reactive protein, crp, acute coronary, inflammatory biomarkers, open label trial

\section{Introduction}

Over the past few years, high-sensitivity C-reactive protein (hs-CRP) has emerged out to be a very useful and reliable clinical marker of cardiovascular (CV) risk. It is an acute phase reactant and has been predicting both primary and secondary risks of a $\mathrm{CV}$ event. It has also been suggested in the research that elevated levels of inflammatory biomarkers - hs-CRP and erythrocyte sedimentation rate (ESR) - point towards subclinical atherosclerosis long before a major acute coronary event occurs [1-2].

In a recent meta-analysis, including patients with acute coronary syndrome (ACS), it was deduced that patients with moderately elevated hs-CRP $(3.1-10 \mathrm{mg} / \mathrm{dL})$ and severely elevated $\mathrm{hs}-\mathrm{CRP}(>10 \mathrm{mg} / \mathrm{dL})$ have 1.40 times and 2.18 times greater long-term risk of recurrent $\mathrm{CV}$ events or even death respectively [3]. HsCRP has not only been regarded as a predictor of adverse cardiovascular outcomes and atherosclerosis but also as a mediator. Hs-CRP has been established to play a critical role in all steps of atherogenesis such as complement activation, activity of macrophages, inflammatory cytokine release, tissue factor induction, endothelial dysfunction, and production of nitric oxide [4].

The largest trial conducted to study the effect of statins on hs-CRP levels was the JUPITER study (Justification for the Use of Statins in Prevention: an Intervention Trial Evaluating Rosuvastatin). With $20 \mathrm{mg}$ rosuvastatin, hs-CRP levels were reduced by $37 \%$. Reduction in hs-CRP levels significantly reduced the risk of all primary major cardiovascular events including myocardial infarction (MI), stroke, arterial revascularization, hospitalization for unstable angina (UA), or death from cardiovascular causes [5]. Since then, research has been focused on the role of statins and other agents in reducing hs-CRP levels [6].

Statins impart pleiotropic effects - that is, they are simultaneously capable of producing more than one benefit, and in cardiovascular risk reduction, they accomplish more than simply lowering cholesterol on the vasculature which contributes to their anti-anginal and anti-ischemic traits. Statins stop the inflammatory process in the endothelium of vessel walls through molecular pathways, involving both innate and adaptive immune systems. Statins inhibit the mevalonate pathway and isoprenoid formation, resulting in increased availability of nitric oxide and protection from ischemia-reperfusion injury. Statins also improve endothelial function, enhance ischemic vasodilatory response, and modulate inflammation [7-8]. Rosuvastatin and atorvastatin are both high-intensity statins and have shown great results in lowering low-density lipoprotein (LDL) which is primarily responsible for all adverse outcomes of hyperlipidemia [9]. Randomized trials, as well as meta-analyses, have shown superior effects of rosuvastatin in reverting coronary atherosclerotic plaques and reduction of hs-CRP levels [10-11]. However, to the best of our knowledge, no such comparative trial has been published from Pakistan to discuss the superior statin in the South Asian

\section{How to cite this article}

Kumar B, Shah M, Kumar R, et al. (June 14, 2019) Comparison of Atorvastatin and Rosuvastatin in Reduction of Inflammatory Biomarkers in Patients with Acute Coronary Syndrome. Cureus 11(6): e4898. 


\section{Cureus}

population. The aim of this study is to compare the effects of rosuvastatin and atorvastatin in lowering hsCRP levels in statin-naive patients admitted with acute coronary syndrome.

\section{Materials And Methods}

We conducted a prospective, open-label, randomized trial in the cardiovascular unit of a tertiary care hospital in Pakistan from January till December 2018. We took consent from all patients, and the study was approved by the ethical review committee of the institute. In order to compare the effects of atorvastatin and rosuvastatin on the inflammatory markers in patients of ACS, we included adult patients of age 18 years and above, of both genders, diagnosed with STEMI, NSTEMI, or UA according to World Health Organization criteria [12] who were not taking statins.

We excluded patients who were taking either statins and/or any other drug which lower serum lipid levels, patients with a history of statin hypersensitivity, patients in whom statins were contraindicated, patients with severe cardiac dysfunction (ejection fraction < 30\%), and any other comorbidity including severe anemia and hepatic or renal failure. Patients who were surgically managed were also excluded. We also excluded pregnant or lactating women.

After fulfilling the inclusion criteria and signing the informed consent, the patients were randomized into two groups. Group A received $40 \mathrm{mg}$ rosuvastatin daily and group B received $20 \mathrm{mg}$ atorvastatin daily along with their standard regime which included aspirin, clopidogrel, beta-blocker, nitrates, and an angiotensinconverting enzyme inhibitor. Serum lipid profile, hs-CRP, and ESR were recorded for all patients at baseline (before starting therapy) and then again after four weeks. Lipid profile was measured using Vitros 250 automatic analyzer (Ortho Clinical Diagnostics, Raritan, NJ). Hs-CRP levels were measured using Turbox hsCRP kit (for protein analyzer Turbox plus) by turbidimetry method. ESR was measured using Westergren method.

Data was entered and analyzed using the IBM SPSS Statistics for Windows, Version 22 (IBM Corp., Armonk, $\mathrm{NY}$ ). Mean and standard deviation were calculated for continuous variables including lipid profile, hs-CRP, and ESR levels for groups A and B. We correlated the means within each group (baseline vs. four weeks) by applying dependent T-test and within the two groups (at four weeks of group A vs. group B) by applying independent T-test. P value $\leqslant 0.05$ was taken as significant.

\section{Results}

At baseline there were 104 patients in group A and 103 in group B. By four weeks, there were 99 patients in group A and 94 in group B. The rest were lost to follow-up.

The changes in hs-CRP levels in both groups during the study period is shown below in Table 1 .

\begin{tabular}{|c|c|c|c|c|c|}
\hline Groups & At Baseline & At Four Weeks & Mean change $(\%)$ & P-value* & P-value ${ }^{\star \star}$ \\
\hline Group A & $38.34 \pm 10.23$ & $18.46 \pm 6.35$ & $51.85 \pm 3.79$ & $<0.0001$ & \multirow{2}{*}{$<0.0001$} \\
\hline Group B & $38.19 \pm 12.38$ & $24.67 \pm 8.45$ & $35.40 \pm 3.17$ & $<0.0001$ & \\
\hline
\end{tabular}

TABLE 1: Mean change in hs-CRP levels ( $\mathrm{mg} / \mathrm{L}$ ) during the study period

Group A: Rosuvastatin 40 mg daily

Group B: Atorvastatin $20 \mathrm{mg}$ daily

* In each group compared at four weeks from baseline

** Inter-group comparison at four weeks

With four weeks of treatment, both rosuvastatin and atorvastatin showed a statistically significant reduction in serum hs-CRP levels $(\mathrm{p}<0.0001)$. In the rosuvastatin group, there was a mean $51 \%$ decrease in serum hsCRP levels; in the atorvastatin group, there was a mean $35 \%$ decrease. In inter-group comparison, the rosuvastatin group showed markedly low serum hs-CRP levels than the atorvastatin group after four weeks of therapy $(18.46 \pm 6.35$ vs. $24.67 \pm 8.45)(\mathrm{p}<0.0001)$.

The changes in ESR levels in both groups during the study period is shown below in Table 2 .

\begin{tabular}{|c|c|c|c|c|c|}
\hline Groups & At Baseline & At Four Weeks & Mean change (\%) & P-value* & P-value ${ }^{* *}$ \\
\hline Group A & $23.47 \pm 13.53$ & $19.59 \pm 11.83$ & $16.53 \pm 1.25$ & 0.031 & \multirow{2}{*}{$<0.0001$} \\
\hline Group B & $23.88 \pm 11.09$ & $20.52 \pm 12.13$ & $14.07 \pm 0.93$ & 0.043 & \\
\hline
\end{tabular}

TABLE 2: Mean change in ESR levels $(\mathrm{mm} / \mathrm{Hr})$ during the study period

Group A: Rosuvastatin $40 \mathrm{mg}$ daily

Group B: Atorvastatin $20 \mathrm{mg}$ daily

* In each group compared at four weeks from baseline

** Inter-group comparison at four weeks 


\section{Cureus}

With four weeks of treatment, both rosuvastatin $40 \mathrm{mg}$ group and atorvastatin $20 \mathrm{mg}$ group showed a statistically significant reduction in ESR levels ( $\mathrm{p}<0.05$ ). In the rosuvastatin $20 \mathrm{mg}$ group, there was a mean $16 \%$ decrease in ESR levels and in the atorvastatin $40 \mathrm{mg}$ group, there was a mean 14\% decrease. In intergroup comparison, the rosuvastatin $20 \mathrm{mg}$ group showed lower ESR levels than the atorvastatin $40 \mathrm{mg}$ group after four weeks of therapy $(19.59 \pm 11.83$ vs. $20.52 \pm 12.13)$. The difference was statistically significant $(\mathrm{p}<0.0001)$.

The mean change in lipid profile in both groups during the study period is shown below in Table 3 .

\begin{tabular}{|c|c|c|c|c|c|c|}
\hline Groups & Lipid Profile & At Baseline & At Four Weeks & Mean change (\%) & P-value* & P-value ${ }^{* x}$ \\
\hline \multirow{5}{*}{ Group A } & TC & $223.43 \pm 33.56$ & $149.56 \pm 26.78$ & $73.87 \pm 6.78$ & $<0.0001$ & 0.301 \\
\hline & HDL & $38.78 \pm 9.48$ & $41.02 \pm 9.56$ & $5.77 \pm 0.08$ & 0.09 & 0.523 \\
\hline & LDL & $198.36 \pm 53.66$ & $66.11 \pm 21.84$ & $66.67 \pm 5.92$ & $<0.0001$ & 0.270 \\
\hline & VLDL & $51.11 \pm 29.38$ & $34.16 \pm 16.23$ & $33.16 \pm 4.47$ & $<0.0001$ & 0.01 \\
\hline & TG & $143.60 \pm 53.39$ & $129.23 \pm 46.74$ & $10.00 \pm 1.24$ & 0.04 & 0.02 \\
\hline \multirow{5}{*}{ Group B } & TC & $220.91 \pm 35.84$ & $153.56 \pm 26.78$ & $30.48 \pm 2.52$ & $<0.0001$ & \\
\hline & HDL & $39.28 \pm 10.54$ & $40.17 \pm 8.87$ & $2.21 \pm 1.58$ & 0.524 & \\
\hline & LDL & $200.49 \pm 54.14$ & $70.87 \pm 36.57$ & $64.65 \pm 3.24$ & $<0.0001$ & \\
\hline & VLDL & $50.56 \pm 28.53$ & $39.27 \pm 12.34$ & $22.32 \pm 5.67$ & 0.0005 & \\
\hline & TG & $145.07 \pm 48.81$ & $115.43 \pm 36.98$ & $20.43 \pm 2.42$ & $<0.0001$ & \\
\hline
\end{tabular}

TABLE 3: Mean change in lipid profile $(\mathrm{mg} / \mathrm{dL})$ during the study period

Abbreviations: TC, total cholesterol; HDL, high-density cholesterol; LDL, low-density cholesterol; VLDL, very low-density cholesterol; TG, triglycerides.

Group A: Rosuvastatin $40 \mathrm{mg}$ daily

Group B: Atorvastatin $20 \mathrm{mg}$ daily

* In each group compared at four weeks from baseline

As seen in Table 3, in both rosuvastatin and atorvastatin groups, a mean favorable change was observed in total cholesterol (TC), low density lipoprotein (LDL), very low density lipoprotein (VLDL), and triglycerides (TGs) that was statistically significant over a period of four weeks $(\mathrm{p}<0.05)$. When inter-group comparison was done, only the change in mean VLDL and TGs was statistically significant $(\mathrm{p}<0.05)$.

\section{Discussion}

This study has evaluated that although the lipid-lowering effects of atorvastatin and rosuvastatin are comparable, the latter has a more profound impact on the reduction of pro-inflammatory markers, especially hs-CRP, which is an established predictor of cardiovascular morbidity and mortality. When tested between the groups, rosuvastatin showed significantly lower hs-CRP levels than atorvastatin at the end of the study.

To the best of our knowledge, this is the first published data on the comparison of anti-inflammatory effects of atorvastatin and rosuvastatin from Pakistan. However, this trial was open-label and conducted in only one center which makes its methodology not very robust. Although there has been a reduction in hs-CRP levels at the end of the study, the levels were still higher than the upper normal limit. This deduces that the patients should've followed the treatment for a longer duration to reach the safe limits of hs-CRP. The case with ESR is similar. However, the study couldn't be stretched for a longer duration.

The literature regarding the superiority of either statin in the reduction of pro-inflammatory markers is not concrete. In a randomized open-label trial with diabetic patients, only atorvastatin significantly reduced hsCRP levels ( $\mathrm{p}=0.02$ ) while rosuvastatin did not. There was also no statistically significant difference between the groups [13]. In another randomized double-blind trial, there was a statistically significant reduction in hs-CRP levels with both atorvastatin and rosuvastatin. At 16 weeks, there was a $40 \%$ reduction with rosuvastatin and $34 \%$ reduction with atorvastatin. However, the differences were not statistically significant between the groups [14]. Although JUPITER trial has been a landmark trial in establishing the role of rosuvastatin in protective anti-inflammatory effects against CV risk, it did not compare the two statins or rosuvastatin with any other lipid-lowering agent [5]. In another meta-analysis, rosuvastatin displayed a more pronounced effect in reducing LDL than atorvastatin $(P<0.001)$, but not in increasing HDL $(P=0.22)$ and reducing hs-CRP $(\mathrm{P}=0.68)$ [10]. In another study with obese type 2 diabetic patients, both atorvastatin and rosuvastatin significantly reduced hs-CRP levels; however, the differences were not significant between the groups [15]

Khurana et al. have been substantial in supporting the role of rosuvastatin against inflammatory markers. It was an open-label trial which showed that hs-CRP levels were significantly decreased after four weeks in both rosuvastatin and atorvastatin groups $(\mathrm{P}<0.001)$. Between the groups, the test revealed that the rosuvastatin group showed significantly lower hs-CRP levels as compared to the atorvastatin group $(\mathrm{P}<0.05)$. The mean percentage decrease in hs-CRP after four weeks in the rosuvastatin group was $44 \%$ and that in atorvastatin group was $35 \%$ [16]. In another recent study with ACS patients, both atorvastatin $80 \mathrm{mg}$ and rosuvastatin $40 \mathrm{mg}$ were effective in causing a statistically significant reduction in hs-CRP levels. Rosuvastatin was more effective in reducing hs-CRP levels than atorvastatin [17]. 


\section{Cureus}

Rosuvastatin was also studied as pre-treatment before percutaneous coronary intervention (PCI) following myocardial infarction. After a mean follow-up of 12 months, a major cardiovascular event (MACE) occurred in $20 \%$ controls (not receiving rosuvastatin) as compared to $9 \%$ in the rosuvastatin group $(P<0.05)$. Hs-CRP levels were less elevated in the rosuvastatin group than in the control group at 24 hours after PCI. Rosuvastatin loading was an independent predictor of a reduction in the risk of MACEs at 12 months [18].

Elevated hs-CRP has been established as a prognostic indicator of new MACE and mortality in patients with ACS [19]. Effective medical intervention to control this underlying micro-inflammation has a critical role in preventing mortality and bettering disease outcome. Statins have shown striking results in reducing hs-CRP levels in patients with ACS. While the choice of statin has been controversial with some literature supporting atorvastatin and other supporting rosuvastatin; the role of rosuvastatin has been more beneficial.

Longitudinal double-blind randomized trials are recommended to follow ACS patients until hs-CRP levels return to normal, and to assess the impact of statin choice in all-cause mortality, cardiac mortality, and new MACEs.

\section{Conclusions}

In statin-naïve patients admitted with acute coronary syndrome, the role of atorvastatin and rosuvastatin has been comparable in optimizing the lipid profile. Both groups showed a statistically significant reduction in ESR and hs-CRP levels at four weeks within groups as well as between groups. Rosuvastatin showed a $50 \%$ decrease in serum hs-CRP levels and atorvastatin showed a 35\% reduction. Rosuvastatin has a more effective role in reducing micro-inflammation in ACS patients which helps improve the disease outcome.

\section{Additional Information}

\section{Disclosures}

Human subjects: Consent was obtained by all participants in this study. Jinnah Postgraduate Medical Centre issued approval MU/ECA/18/244. Animal subjects: All authors have confirmed that this study did not involve animal subjects or tissue. Conflicts of interest: In compliance with the ICMJE uniform disclosure form, all authors declare the following: Payment/services info: All authors have declared that no financial support was received from any organization for the submitted work. Financial relationships: All authors have declared that they have no financial relationships at present or within the previous three years with any organizations that might have an interest in the submitted work. Other relationships: All authors have declared that there are no other relationships or activities that could appear to have influenced the submitted work.

\section{References}

1. Thomas MR, Lip GY: Novel risk markers and risk assessments for cardiovascular disease . Circ Res. 2017, 120:133-49. 10.1161/CIRCRESAHA.116.309955

2. Eapen DJ, Manocha P, Patel RS, et al.: Aggregate risk score based on markers of inflammation, cell stress, and coagulation is an independent predictor of adverse cardiovascular outcomes. J Am Coll Cardiol. 2013, 62:329-37. 10.1016/j.jacc.2013.03.072

3. He LP, Tang XY, Ling WH, Chen WO, Chen YM: Early C-reactive protein in the prediction of long-term outcomes after acute coronary syndromes: a meta-analysis of longitudinal studies. Heart. 2010, 96:339-46. 10.1136/hrt.2009.174912

4. Shrivastava AK, Singh HV, Raizada A, Singh SK: C-reactive protein, inflammation and coronary heart disease. Egypt Heart J. 2015, 67:89-97. 10.1016/j.ehj.2014.11.005

5. Ridker PM, Danielson E, Fonseca FA, et al.: Rosuvastatin to prevent vascular events in men and women with elevated C-reactive protein. N Engl J Med. 2008, 359:2195-207. 10.1056/NEIMoa0807646

6. Koenig W: High-sensitivity C-reactive protein and atherosclerotic disease: from improved risk prediction to risk-guided therapy. Int J Cardiol. 2013, 168:5126-34. 10.1016/j.ijcard.2013.07.113

7. Tousoulis D, Psarros C, Demosthenous M, Patel R, Antoniades C, Stefanadis C: Innate and adaptive inflammation as a therapeutic target in vascular disease: the emerging role of statins. J Am Coll Cardiol. 2014, 63:2491-502. 10.1016/j.jacc.2014.01.054

8. Lardizabal JA, Deedwania PC: The anti-ischemic and anti-anginal properties of statins . Curr Atheroscler Rep. 2011, 13:43-50. 10.1007/s11883-010-0147-y

9. Zhang L, Zhang S, Yu Y, Jiang H, Ge J: Efficacy and safety of rosuvastatin vs. atorvastatin in lowering LDL cholesterol: A meta-analysis of trials with East Asian populations. Herz. 2018, 10.1007/s00059-018-4767-2

10. Qian C, Wei B, Ding I, Wu H, Cai X, Li B, Wang Y: Meta-analysis comparing the effects of rosuvastatin versus atorvastatin on regression of coronary atherosclerotic plaques. Am J Cardiol. 2015, 116:1521-6. 10.1016/j.amjcard.2015.08.010

11. Ma Q, Zhou Y, Zhai G, et al.: Meta-analysis comparing rosuvastatin and atorvastatin in reducing concentration of C-reactive protein in patients with hyperlipidemia. Angiology. 2016, 67:526-35. $10.1177 / 0003319715599863$

12. Nomenclature and criteria for diagnosis of ischemic heart disease. Report of the Joint International Society and Federation of Cardiology/World Health Organization task force on standardization of clinical nomenclature. Circulation. 1979, 59:607-9. 10.1161/01.CIR.59.3.607

13. Anagnostis $P$, Adamidou F, Slavakis A, et al.: Comparative effect of atorvastatin and rosuvastatin on $25-$ hydroxy-vitamin D levels in non-diabetic patients with dyslipidaemia: a prospective randomized open-label pilot study. Open Cardiovasc Med J. 2014, 8:55-60. Accessed: June 11, 2019: 10.2174/187419240140801005510.2174/187419240140801005510.2174/187419240140801005510.2174/187419240140801005510.2174/1874192

14. Betteridge DJ, Gibson JM, Sager PT: Comparison of effectiveness of rosuvastatin versus atorvastatin on the achievement of combined C-reactive protein $(<2 \mathrm{mg} / \mathrm{L})$ and low-density lipoprotein cholesterol $(<70 \mathrm{mg} / \mathrm{dl})$ targets in patients with type 2 diabetes mellitus (from the ANDROMEDA study). Am J Cardiol. 2007, 100:1245-8. 10.1016/.amjcard.2007.05.044

15. Sindhu S, Singh HK, Salman MT, Fatima J, Verma VK: Effects of atorvastatin and rosuvastatin on highsensitivity C-reactive protein and lipid profile in obese type 2 diabetes mellitus patients. J Pharmacol Pharmacother. 2011, 2:261-5. 10.4103/0976-500X.85954

16. Khurana S, Gupta S, Bhalla H, Nandwani S, Gupta V: Comparison of anti-inflammatory effect of atorvastatin with rosuvastatin in patients of acute coronary syndrome. J Pharmacol Pharmacother. 2015, 6:130-5. 10.4103/0976-500X.162011

17. George M, Joseph L, Chacko BK, Jose J: A comparative study on the effect of HMG-CoA reductase inhibitor on C-reactive protein in patients with acute coronary syndrome. World J Pharm Res. 2017, 6:1852-63. 10.20959/wjpr20178-9101

18. Yun KH, Oh SK, Rhee SJ, Yoo NJ, Kim NH, Jeong JW: 12-month follow-up results of high dose rosuvastatin loading before percutaneous coronary intervention in patients with acute coronary syndrome. Int I Cardiol. 


\section{Cureus}

2011, 146:68-72. 10.1016/j.ijcard.2010.04.052

19. Nordenskjöld AM, Baron T, Eggers KM, Jernberg T, Lindahl B: Predictors of adverse outcome in patients with myocardial infarction with non-obstructive coronary artery (MINOCA) disease. Int J Cardiol. 2018, 261:18-23. 10.1016/.ijcard.2018.03.056 